\title{
DEFORMATION QUANTIZATION VIA FELL BUNDLES
}

\author{
BEATRIZ ABADIE* and RUY EXEL**
}

\begin{abstract}
A method for deforming $C^{*}$-algebras is introduced, which applies to $C^{*}$-algebras that can be described as the cross-sectional $C^{*}$-algebra of a Fell bundle. Several well known examples of non-commutative algebras, usually obtained by deforming commutative ones by various methods, are shown to fit our unified perspective of deformation via Fell bundles. Examples are the non-commutative spheres of Matsumoto, the non-commutative lens spaces of Matsumoto and Tomiyama, and the quantum Heisenberg manifolds of Rieffel. In a special case, in which the deformation arises as a result of an action of $\mathrm{R}^{2 d}$, assumed to be periodic in the first $d$ variables, we show that we get a strict deformation quantization.
\end{abstract}

\section{Introduction}

One of the most popular methods for constructing deformations of $C^{*}$-algebras is to describe the given $C^{*}$-algebra by means of generators and relations, and, after introducing a deformation parameter into these relations, to consider the universal $C^{*}$-algebra for the new relations. This procedure can be used, for example, for constructing the non-commutative torus [14], the soft torus [5], the quantum $S U_{2}$ groups [19], the non-commutative spheres [10], the noncommutative lens spaces [12], and the algebra of the $q$-canonical commutation relations [9].

However, $C^{*}$-algebras arising from generators and relations are often intractable objects, motivating one to search for alternative constructions. The goal of the present work is to show how the techniques of Fell bundles (also known as $C^{*}$-algebraic bundles [8]) apply to the study of deformations of $C^{*}$-algebras.

Since our techniques apply to $C^{*}$-algebras that can be expressed as the cross-sectional $C^{*}$-algebra of a Fell bundle over a group $G$, the first step in our construction consists of finding such a description of the algebra to be deformed.

The second step, described in Section 2, makes use of an action $\theta$ of the group $G$ on $\mathscr{B}$ to deform the Fell bundle structure, by introducing a new

\footnotetext{
* Partially supported by CONICYT, Proyecto 2002 - Uruguay.

** Partially supported by CNPq - Brazil.

Received August 11, 1997; in revised form October 15, 1998.
} 
multiplication and a new involution without changing the norm and the linear structure of $\mathscr{B}$. The deformed algebra is then obtained by taking the crosssectional $C^{*}$-algebra of the deformed Fell bundle. The invariance of the linear structure and the norm of the Fell bundle under the deformation allows us to embed part of the original algebra into its deformed version.

In section 3 we show that, when a family $\left\{\theta^{\hbar}\right\}_{\hbar \in I}$ of actions of $G$ on $\mathscr{B}$ as above is given, that satisfies some continuity conditions on the interval $I$ of real numbers, then the family of deformed $C^{*}$-algebras is a continuous field of $C^{*}$-algebras. We restrict our discussion to the case of discrete groups, which is considerably easier to handle from a technical point of view, and covers all of our applications. These continuity results are, essentially, reworkings of Rieffel's ideas in [15] for our more general situation of Fell bundles.

We discuss in section 4 a situation that enables us to carry out simultaneously the two steps described above. That is the case when $G$ is an abelian discrete group, and $\theta$ and $\gamma$ are commuting actions of $G$ and its dual $\widehat{G}$, respectively, on a $C^{*}$-algebra $B$. Then, by means of the action $\gamma, B$ can be described as the cross-sectional $C^{*}$-algebra of a Fell bundle over $G$, while the action $\theta$ provides the setting for the deformation.

This approach essentially consists of introducing a deformation parameter after taking a certain Fourier transform, a method that has already been used by other authors, as Rieffel (see, for example, the formula for the definition of $*_{\hbar}$ on page 541 of [16]). The advantage of emphasizing the Fell bundle structure is, perhaps, in making some formulas more transparent.

This rather elementary construction provides some interesting examples, which we present in sections 6,7 , and 8 . We show that the non-commutative spheres, the non-commutative lens spaces, and the quantum Heisenberg manifolds [16], can all be seen under this unified perspective.

Following Rieffel's approach ([17], [18]), we discuss in section 5 the case of a $C^{*}$-algebra $B$ carrying an action of $\mathrm{T}^{d} \times \mathrm{R}^{d}$, T being the unit circle. This situation yields the setting to construct, by our methods, a deformation $\left\{B^{(\hbar)}\right\}$ of $B$, and we compute the derivative at zero of the deformed multiplication as a function of the deformation parameter $\hbar$.

As Rieffel mentions in page 84 of [18], in this particular case, where one benefits from the compactness of $\mathrm{T}^{d}$, the $C^{*}$-algebras involved are cross-sectional $C^{*}$-algebras of a Fell bundle. Rieffel's deformation may then be seen as a deformation of the Fell bundle structure by means of a 2-cocycle. However, our approach differs from Rieffel's because our deformation is caused by a group action, instead of a 2-cocycle, and because we deform both the multiplication and the involution, while Rieffel's deformation affects only the former.

The computation of the derivative is initially done for a very restrictive class of elements $f$ and $g$ in $B$, namely the smooth elements belonging, each, 
to a spectral subspace for $\gamma$. The proof of this result is extremely simple and the formulas involved show, in a very transparent way, the roles of the various ingredients present in the context. In particular, the heavy machinery of oscillatory integrals of [17] does not intervene, thanks, of course, to the simplification introduced by the periodicity assumption. The formula for the derivative of the deformed multiplication, above, is then extended to smooth elements $f$ and $g$.

Combining this with the fact that the $B^{(\hbar)}$ form a continuous field of $C^{*}$-algebras, we get a strict deformation quantization in the sense of Rieffel [17], [18].

The authors would like to acknowledge the support of CONICYT (Uruguay) and FAPESP (Brazil) for funding numerous academic visits while this research was conducted.

\section{The Deformation}

Let $G$ be a locally compact topological group and let $\mathscr{B}=\left\{B_{t}\right\}_{t \in G}$ be a $C^{*}$ algebraic bundle over $G$. The reader is referred to [8] for a comprehensive treatment of the basic theory of $C^{*}$-algebraic bundles. These objects have recently been referred to as "Fell bundles", a terminology we have chosen to adopt. In what follows, we shall identify $\mathscr{B}$ with the total bundle space $\bigcup_{t \in G} B_{t}$.

Let $\mathscr{D}=\left\{D_{t}\right\}_{t \in G}$ be another Fell bundle over $G$. In the spirit of [8, VIII.3.3], a map $\psi$ from $\mathscr{B}$ to $\mathscr{D}$ is called a homomorphism if

i) $\psi$ is continuous,

ii) $\psi\left(B_{t}\right) \subseteq D_{t}$, for all $t$ in $G$,

iii) $\psi$ is linear on each $B_{t}$,

iv) $\psi(a b)=\psi(a) \psi(b)$, for all $a, b$ in $\mathscr{B}$, and

v) $\psi\left(a^{*}\right)=\psi(a)^{*}$, for all $a$ in $\mathscr{B}$.

Let $\psi$ be a homomorphism from $\mathscr{B}$ to $\mathscr{D}$. Since $\psi$ restricts to a $*$-homomorphism between the $C^{*}$-algebras $B_{e}$ and $D_{e}$ ( $e$ being the unit group element), it is contractive there. Also, for each $b_{t}$ in $B_{t}$ we have

$$
\left\|\psi\left(b_{t}\right)\right\|^{2}=\left\|\psi\left(b_{t}^{*} b_{t}\right)\right\| \leq\left\|b_{t}^{*} b_{t}\right\|=\left\|b_{t}\right\|^{2},
$$

so that $\psi$ is in fact norm-contractive on $\mathscr{B}$.

If $\psi$ is bijective, then $\psi^{-1}$ is continuous as well [8, II.13.17], so it is also a homomorphism, and $\psi$ is isometric. In this case we say that $\psi$ is an isomorphism (an automorphism of $\mathscr{B}$, when $\mathscr{D}=\mathscr{B}$ ).

Given another locally compact topological group $H$, an action of $H$ on $\mathscr{B}$ is a group homomorphism $\theta: H \rightarrow \operatorname{Aut}(\mathscr{B})$. The action $\theta$ is said to be continuous if so is the map $(x, b) \in H \times \mathscr{B} \longmapsto \theta_{x}(b) \in \mathscr{B}$. 
Let us now suppose we are given a Fell bundle $\mathscr{B}=\left\{B_{t}\right\}_{t \in G}$ over the locally compact group $G$, as well as a continuous action $\theta$ of the same group $G$ on $\mathscr{B}$. We wish to construct a new product on $\mathscr{B}$, denoted $\times$, and a new involution, called ${ }^{\diamond}$, providing a "deformed" bundle structure. In order to do so, define for $a_{t}$ in $B_{t}$ and $b_{s}$ in $B_{s}$,

$$
a_{t} \times b_{s}=a_{t} \theta_{t}\left(b_{s}\right), \text { and } a_{t}^{\diamond}=\theta_{t}^{-1}\left(a_{t}^{*}\right) .
$$

Proposition 2.1. If $\mathscr{B}$ keeps its linear, topological and norm structure, but is given the deformed operations $\times$ and ${ }^{\diamond}$, then it is a Fell bundle.

Proof. To check that the new multiplication operation is continuous, we shall use [8, VIII.2.4]. That is, given continuous sections $\beta$ and $\gamma$ of $\mathscr{B}$, we must show that the map $(r, s) \in G \times G \longmapsto \beta(r) \times \gamma(s) \in \mathscr{B}$ is continuous. Now, we have $\beta(r) \times \gamma(s)=\beta(r) \theta_{r}(\gamma(s))$, which is continuous by the continuity of $\theta$ and of the original multiplication. A similar argument shows that the deformed involution is continuous.

Let us now verify the associativity of $\times$. Given $a_{r}$ in $B_{r}, b_{s}$ in $B_{s}$, and $c_{t}$ in $B_{t}$ we have

$$
\begin{gathered}
\left(a_{r} \times b_{s}\right) \times c_{t}=\left(a_{r} \theta_{r}\left(b_{s}\right)\right) \times c_{t}=a_{r} \theta_{r}\left(b_{s}\right) \theta_{r s}\left(c_{t}\right) \\
=a_{r} \theta_{r}\left(b_{s} \theta_{s}\left(c_{t}\right)\right)=a_{r} \times\left(b_{s} \theta_{s}\left(c_{t}\right)\right)=a_{r} \times\left(b_{s} \times c_{t}\right) .
\end{gathered}
$$

As for the anti-multiplicativity of the involution, let $a_{r} \in B_{r}$ and $b_{s} \in B_{s}$. Then

$$
\begin{gathered}
\left(a_{r} \times b_{s}\right)^{\diamond}=\left(a_{r} \theta_{r}\left(b_{s}\right)\right)^{\diamond}=\theta_{(r s)^{-1}}\left(a_{r} \theta_{r}\left(b_{s}\right)\right)^{*}=\theta_{s^{-1}} \theta_{r^{-1}}\left(\theta_{r}\left(b_{s}^{*}\right) a_{r}^{*}\right) \\
=\theta_{s^{-1}}\left(b_{s}^{*}\right) \theta_{s^{-1}} \theta_{r^{-1}}\left(a_{r}^{*}\right)=\theta_{s^{-1}}\left(b_{s}^{*}\right) \times \theta_{r^{-1}}\left(a_{r}^{*}\right)=b_{s}^{\diamond} \times a_{r}^{\diamond} .
\end{gathered}
$$

The verification of the remaining axioms is routine.

Definition 2.2. The bundle constructed above, denoted by $\mathscr{B}^{\theta}$, will be called the $\theta$-deformation of $\mathscr{B}$.

Recall that a Fell bundle is said to be saturated [8, VIII.2.8] if $B_{r s}=B_{r} B_{s}$ (closed linear span) for all $r, s$. In the special case that $G$ is equipped with a "length" function $|\cdot|: G \rightarrow \mathrm{R}_{+}$satisfying $|e|=0$, and the triangular inequality $|r s| \leq|r|+|s|$, then we say that $\mathscr{B}$ is semi-saturated (see $[6,4.1$, 4.8], [7, 6.2]), if $B_{r s}=B_{r} B_{s}$, whenever $r, s \in G$ are such that $|r s|=|r|+|s|$.

Proposition 2.3. If $\mathscr{B}$ is saturated (resp. semi-saturated) then so is $\mathscr{B}^{\theta}$.

PROOF. It is enough to observe that $B_{r} \times B_{s}=B_{r} \theta_{r}\left(B_{s}\right)=B_{r} B_{s}$. 


\section{Continuous fields arising from deformations}

The purpose of this section is to show that the collection of deformed algebras, originated from a continuous family of group actions on a Fell bundle, gives rise to a continuous field of $C^{*}$-algebras.

We first establish some facts on Fell bundles over discrete groups that will enable us to extend the techniques in [15] to discuss upper semicontinuity. Let $\mathscr{B}$ and $\mathscr{D}$ be fell bundles over a discrete group $G$, and let $\Phi: \mathscr{D} \rightarrow \mathscr{B}$ be a Fell bundle homomorphism. Since $\Phi$ is contractive, one can define $\Phi^{1}: L^{1}(\mathscr{D}) \rightarrow$ $L^{1}(\mathscr{B})$ by $[\Phi(f)](x)=\Phi[f(x)]$, for $f \in L^{1}(\mathscr{D})$, and $x \in G$. It is easily checked that $\Phi^{1}$ is a $*$-algebra homomorphism, so it gives rise to a $C^{*}$-algebra homomorphism $\tilde{\Phi}: C^{*}(\mathscr{D}) \rightarrow C^{*}(\mathscr{B})$.

A sequence of Fell bundle homomorphisms

$$
0 \rightarrow \mathscr{E} \stackrel{i}{\rightarrow} \mathscr{D} \stackrel{\Pi}{\rightarrow} \mathscr{B} \rightarrow 0
$$

is said to be exact if so are the sequences

$$
0 \rightarrow \mathscr{E}_{x} \stackrel{i \mid \mathscr{E}_{x}}{\rightarrow} \mathscr{D}_{x} \stackrel{\Pi \mid \mathscr{O}_{x}}{\rightarrow} \mathscr{B}_{x} \rightarrow 0
$$

for all $x \in G$.

Lemma 3.1. Let $0 \rightarrow \mathscr{E} \stackrel{i}{\rightarrow} \mathscr{D} \stackrel{\Pi}{\rightarrow} \mathscr{B} \rightarrow 0$ be an exact sequence of Fell bundle homomorphisms over a discrete group $G$. Then $0 \rightarrow C^{*}(\mathscr{E}) \stackrel{\tilde{i}}{\rightarrow}$ $C^{*}(\mathscr{D}) \stackrel{\tilde{\Pi}}{\rightarrow} C^{*}(\mathscr{B}) \rightarrow 0$ is also exact.

Proof. In view of $[20,2.29]$, and [8, VIII 5.11, 16.3], we only need to show that $0 \rightarrow L^{1}(\mathscr{E}) \stackrel{i^{1}}{\rightarrow} L^{1}(\mathscr{D}) \stackrel{\Pi^{1}}{\rightarrow} L^{1}(\mathscr{B}) \rightarrow 0$ is exact. It is apparent from the definition that $i^{1}$ is injective, and that $\operatorname{Im}\left(i^{1}\right)=\operatorname{ker}\left(\Pi^{1}\right)$, so we need only show that $\Pi^{1}$ is onto. Fix $b_{x} \in \mathscr{B}_{x}$ and $\epsilon>0$. Since $b_{x} \delta_{x} \in \operatorname{Im} \tilde{\Pi}$, there exists $\tilde{d} \in C^{*}(\mathscr{D})$ such that $\tilde{\Pi}(\tilde{d})=b_{x} \delta_{x}$, and

$$
\|\tilde{d}\|_{C^{*}(\mathscr{D})} \leq\|\tilde{d}+\operatorname{ker} \tilde{\Pi}\|_{C^{*}(\mathscr{D}) / \operatorname{ker} \tilde{\Pi}}+\epsilon=\left\|b_{x} \delta_{x}\right\|_{C^{*}(\mathscr{B})}+\epsilon=\left\|b_{x}\right\|_{\mathscr{B}_{x}}+\epsilon .
$$

Let $P_{x}^{\mathscr{D}}$ (resp. $\left.P_{x}^{\mathscr{B}}\right)$ denote the projection onto the $x^{\text {th }}$ spectral subspace of $\mathscr{D}($ resp. $\mathscr{B})$. Then $P_{x}^{\mathscr{B}} \tilde{\Pi}=\tilde{\Pi} P_{x}^{\mathscr{D}}$, since the equality holds when restricted to $L^{1}(\mathscr{D})$. Now set $d=P_{x}^{\mathscr{D}}(\tilde{d})$. Then $d \in \mathscr{D}_{x}, \tilde{\Pi}(d)=\tilde{\Pi} P_{x}^{\mathscr{D}}(\tilde{d})=P_{x}^{\mathscr{B}} \tilde{\Pi}(\tilde{d})=$ $b_{x}$, and $\|d\|_{\mathscr{D}_{x}} \leq\|\tilde{d}\|_{C^{*}(\mathscr{D})} \leq\left\|b_{x}\right\|_{\mathscr{B}_{x}}+\epsilon$.

Now, if $\sum b_{n} \delta_{x_{n}} \in L^{1}(\mathscr{B})$, choose as above, for each positive integer $n, d_{n} \in$ $\mathscr{D}_{x_{n}}$ so that $\Pi\left(d_{n}\right)=b_{n}$, and $\left\|d_{n}\right\|_{\mathscr{D}_{x_{n}}} \leq\left\|b_{n}\right\|_{\mathscr{B}_{x_{n}}}+n^{-2}$. Then $\Pi^{1}\left(\sum c_{n} \delta_{x_{n}}\right)=$ $\sum b_{n} \delta_{x_{n}}$. So $\Pi^{1}$ is onto. 
Back to the setting of the previous section, we consider a $C^{*}$-algebra $B$ that can be viewed as the cross-sectional $C^{*}$-algebra of a Fell bundle $\mathscr{B}$ over a discrete group $G$ whose $x^{\text {th }}$ fiber we denote by $B_{x}$. At this point we are ready to get a deformed version of $B$ by means of an action $\theta$ of $G$.

Notice that the algebra $B^{\theta}$ contains as a dense $*$-subalgebra the set $\bigoplus_{x \in G} B_{x}$ of compactly supported cross-sections. Although the $*$-algebra structure of $\bigoplus_{x \in G} B_{x}$ depends on $\theta$, its vector space structure does not.

Our purpose is to produce a continuous field of $C^{*}$-algebras, given a family $\left\{\theta^{\hbar}\right\}$ of actions of $G$ on $\mathscr{B}$. The crucial point is to show that the map $\hbar \mapsto\|\phi\|_{\hbar}$ is continuous for any $\phi \in \bigoplus_{x \in G} B_{x}$, where $\|\phi\|_{\hbar}$ denotes the norm of $\phi$ as an element of $C^{*}\left(\mathscr{B}^{\theta^{\hbar}}\right)$.

Notation 3.2. In the context above, let $I \subset \mathbf{R}$ be an open interval containing 0 and, for each $\hbar \in I$, let $\theta^{\hbar}$ be an action of $G$ on the Fell bundle $\mathscr{B}$ such that $\theta^{0}$ is the identity, and that the map $\hbar \mapsto \theta_{x}^{\hbar}(b)$ is continuous for any fixed $x \in G, b \in \mathscr{B}$. We denote the bundle $\mathscr{B}^{\theta^{\hbar}}$ by $\mathscr{B}^{\hbar}$, and by $\times_{\hbar},{ }^{\diamond_{\hbar}}$ its product and involution, respectively. The norm in $C^{*}\left(\mathscr{B}^{\hbar}\right)$ is denoted by \|\|$_{\hbar}$.

Proposition 3.3. The map $\hbar \mapsto\|\phi\|_{\hbar}$ is upper semicontinuous on I for all $\phi \in \bigoplus_{x \in G} B_{x}$.

Proof. The proof follows the lines of [15]. Let $\mathscr{D}$ be the Fell bundle over $G$ whose $x^{\text {th }}$ fiber is the Banach space $D_{x}=C_{0}\left(I, B_{x}\right)$, with multiplication and involution given by

$$
\left(f_{x} \star f_{y}\right)(\hbar)=f_{x}(\hbar) \times_{\hbar} f_{y}(\hbar), \quad f_{x}^{\star}(\hbar)=\left(f_{x}(\hbar)\right)^{\diamond_{\hbar}},
$$

for $f_{x} \in D_{x}, f_{y} \in D_{y}$. For each $\hbar \in I$ consider the Fell bundle homomorphism $\Pi^{\hbar}: \mathscr{D} \rightarrow \mathscr{B}$, given by $\Pi^{\hbar}(f)=f(\hbar)$. Since $\Pi^{\hbar}$ is onto for any $\hbar \in I$ we get, as in Lemma 3.1, the exact sequence

$$
0 \rightarrow C^{*}\left(\mathscr{E}^{\hbar}\right) \stackrel{\tilde{i}^{\hbar}}{\rightarrow} C^{*}(\mathscr{D}) \stackrel{\tilde{\Pi}^{\hbar}}{\rightarrow} C^{*}\left(\mathscr{B}^{\hbar}\right) \rightarrow 0
$$

where $\mathscr{E}^{\hbar}$ is the Fell bundle whose $x^{\text {th }}$ fiber is $E_{x}^{\hbar}=\operatorname{ker} \Pi_{x}^{\hbar}$, with the structure inherited from $\mathscr{D}$, and $\tilde{i}^{\hbar}$ denotes inclusion.

In order to apply $[15,1.2]$, we next consider $C_{0}(I)$ as a $C^{*}$-subalgebra of the algebra of multipliers of $D_{e}$, in the obvious way, so we can view it ([8, VIII, 3.8]) as a central $C^{*}$-subalgebra of the multiplier algebra of $C^{*}(\mathscr{D})$.

Let $J_{\hbar}=\left\{f \in C_{0}(I): f(\hbar)=0\right\}$. It only remains to show that $C^{*}\left(\mathscr{E}^{\hbar}\right)=$ $C^{*}(\mathscr{D}) J_{\hbar}$. For then, by $[15,1.2]$, we will have that $\hbar \mapsto\left\|\tilde{\Pi}^{\hbar}(\phi)\right\|$ is upper semicontinuous for all $\phi \in C^{*}(\mathscr{D})$. This implies that $\hbar \mapsto\|\psi\|_{\hbar}$ is upper semicontinuous for any $\psi \in \bigoplus_{x \in G} B_{x}$. Now, it is apparent that $\phi j \in L^{1}(\mathscr{E})$ for $j \in J_{\hbar}$, and $\phi \in L^{1}(\mathscr{D})$, which shows that $C^{*}(\mathscr{D}) J_{\hbar} \subset C^{*}\left(\mathscr{E}^{\hbar}\right)$. On the 
other hand, if $\left\{e_{\lambda}\right\}$ is a bounded approximate identity for $J_{\hbar}$, then $\lim _{\lambda} \phi e_{\lambda}=\phi$ for all $\phi \in C^{*}\left(\mathscr{E}^{\dagger}\right)$ : It suffices to show it for compactly supported maps $\phi$, since $\left\{e_{\lambda}\right\}$ is assumed to be bounded. Notice that the statement holds for $\phi=f \delta_{e}$, with $f \in E_{e}^{\hbar}$, because $E_{e}^{\hbar} \cong B_{e} \otimes J_{\hbar}$. Now, if $\phi=f_{x} \delta_{x}$ for some $f_{x} \in E_{x}^{\hbar}$, we have

$$
\left\|\phi e_{\lambda}-\phi\right\|^{2}=\left\|\left(\phi e_{\lambda}-\phi\right)^{*}\left(\phi e_{\lambda}-\phi\right)\right\| \leq\left(\left\|e_{\lambda}\right\|+1\right)\left\|\phi^{*} \phi e_{\lambda}-\phi^{*} \phi\right\|,
$$

which goes to zero because $\phi^{*} \phi \in E_{e}^{\hbar}$. This shows that $C^{*}(\mathscr{D}) J_{\hbar} \supset C^{*}\left(\mathscr{E}^{\hbar}\right)$.

Proposition 3.4. If $G$ is also amenable, then the map $\hbar \mapsto\|\phi\|_{\hbar}$ is lower semicontinuous on I for all $\phi \in \bigoplus_{x \in G} B_{x}$.

Proof. Since $G$ is amenable, the left regular representation $\Lambda^{\hbar}$ of $C^{*}\left(\mathscr{B}^{\hbar}\right)$ is faithful ([7, 2.3 and 4.7]), so it suffices to show that $\hbar \mapsto\left\|\Lambda_{\phi}^{\hbar}\right\|$ is lower semicontinuous for $\phi \in \bigoplus_{x \in G} B_{x}$.

As in [7], for $\hbar \in I$ we denote by $L^{2}\left(\mathscr{B}^{h}\right)$ the completion of $C_{c}\left(\mathscr{B}^{h}\right)$ with its obvious right pre-Hilbert module structure over $B_{e}^{\hbar}$, which yields the norm

$$
\|\xi\|^{2}=\left\|\sum_{x \in G} \xi(x)^{\diamond_{\hbar}} \times_{\hbar} \xi(x)\right\|_{B_{e}^{\hbar}}=\left\|\sum_{x} \theta_{x^{-1}}^{\hbar}\left[\xi(x)^{*} \xi(x)\right]\right\|_{B_{e}^{0}},
$$

for any $\xi \in \bigoplus_{x \in G} B_{x}$, the undecorated involution and multiplication denoting those in $\mathscr{B}^{0}$.

The left regular representation $\Lambda^{\hbar}$ of $\phi \in \bigoplus_{x \in G} B_{x}$ is the adjointable operator given by:

$$
\left(\Lambda_{\phi}^{\hbar} \xi\right)(y)=\sum_{x \in G} \phi(x) \times_{\hbar} \xi\left(x^{-1} y\right)=\sum_{x} \phi(x) \theta_{x}^{\hbar}\left[\xi\left(x^{-1} y\right)\right],
$$

for $\xi \in \bigoplus_{x \in G} B_{x} \subset L^{2}\left(\mathscr{B}^{\hbar}\right)$. So we have

$$
\left\|\Lambda_{\phi}^{\hbar} \xi\right\|_{\hbar}^{2}=\left\|\sum_{x, y} \theta_{y^{-1}}^{\hbar}\left[\left(\phi(x) \theta_{x}^{\hbar}\left(\xi\left(x^{-1} y\right)\right)\right)^{*}\left(\phi(x) \theta_{x}^{\hbar}\left(\xi\left(x^{-1} y\right)\right)\right)\right]\right\|_{B_{e}} .
$$

Notice that the sum above is finite, since both $\phi$ and $\xi$ are compactly supported. Besides, each term of the sum is continuous on $\hbar$, so $\hbar \mapsto\left\|\Lambda_{\phi}^{\hbar} \xi\right\|_{\hbar}$ is continuous. Now fix $\phi \in \bigoplus_{x \in G} B_{x}, \epsilon>0$, and $\hbar_{0} \in I$. Then $\xi_{0} \in C_{c}\left(\mathscr{B}^{h_{0}}\right)$ can be found so that $\left\|x_{0}\right\|=1$ and $\left\|\Lambda_{\phi}^{\hbar_{0}} \xi_{0}\right\|>\left\|\Lambda_{\phi}^{\hbar_{0}}\right\|-\epsilon$. For one can find $\xi \in L^{2}\left(\mathscr{B}^{h_{0}}\right)$ satisfying that inequality for $\frac{\epsilon}{2}$, with $\|\xi\|=1$. Then, given $\left\{\xi_{n}\right\} \subset \bigoplus_{x \in G} B_{x}$ such that $\lim \xi_{n}=\xi$, the sequence $\left\{\frac{1}{\left\|\xi_{n}\right\|} \xi_{n}\right\}$ also converges to $\xi$. So one can take $\xi_{0} \in \bigoplus_{x \in G} B_{x}$, such that $\left\|\xi_{0}\right\|=1$ and $\left\|\xi-\xi_{0}\right\|<\frac{\epsilon}{2}\left\|\Lambda_{\phi}^{\hbar_{0}}\right\|$. Then

$$
\left\|\Lambda_{\phi}^{\hbar_{0}}\right\|-\frac{\epsilon}{2}<\left\|\Lambda_{\phi}^{\hbar_{0}} \xi_{0}\right\|+\frac{\epsilon}{2},
$$


as required. It now follows that, for $\hbar$ close enough to $\hbar_{0}$,

$$
\frac{\left\|\Lambda_{\phi}^{\hbar} \xi_{0}\right\|_{\hbar}}{\left\|\xi_{0}\right\|_{\hbar}}>\left\|\Lambda_{\phi}^{h_{0}}\right\|-\epsilon, \quad \text { so } \quad\left\|\Lambda_{\phi}^{\hbar}\right\|>\left\|\Lambda_{\phi}^{h_{0}}\right\|-\epsilon .
$$

We summarize the previous results in the following theorem.

THEOREM 3.5. Let $\mathscr{B}$ be a Fell bundle over a discrete amenable group $G$, and let $B=C^{*}(\mathscr{B})$. If $\left\{\theta^{\hbar}: \hbar \in I\right\}$ and $\mathscr{B}^{\hbar}$ are as in 3.2, then $\left\{C^{*}\left(\mathscr{B}^{\hbar}\right), \Lambda\right\}$ is a continuous field of $C^{*}$-algebras, such that $C^{*}\left(\mathscr{B}^{0}\right)=B$, where $\Lambda$ is the family of cross-sections obtained, as in [4, 10.2.3], out of $C_{c}\left(\mathscr{B}^{\hbar}\right)$.

\section{Discrete abelian groups}

We would now like to describe a method for producing examples of the above situation. To reduce the technical difficulties to a minimum we will consider here exclusively the case of discrete abelian groups. Several interesting examples, however, will fit this context.

Fix, throughout this section, a discrete abelian group $G$, and let $\widehat{G}$ be its dual, so that $\widehat{G}$ is a compact abelian group. We shall denote the duality between $G$ and $\widehat{G}$ by $(x, t) \in \widehat{G} \times G \longmapsto\langle x, t\rangle \in S^{1}$.

Let $B$ be a $C^{*}$-algebra carrying a continuous action $\gamma$ of $\widehat{G}$. For each $t$ in $G$, the $t$-spectral subspace of $B$ is defined by $B_{t}=\left\{b \in B: \gamma_{x}(b)=\right.$ $\langle x, t\rangle b$, for all $x \in \widehat{G}\}$.

It is easy to check that each $B_{t}$ is a closed linear subspace of $B$, that $B_{r} B_{s} \subseteq$ $B_{r s}$, and that $B_{t}^{*}=B_{t^{-1}}$. By imitating $[6,2.5]$ one can show that $B$ coincides with the closure of $\bigoplus_{t \in G} B_{t}$ (we use the symbol $\bigoplus$ to denote the algebraic direct sum, that is, the set of finite sums) and that the formula

$$
P_{t}(b)=\int_{\widehat{G}}\langle x, t\rangle^{-1} \gamma_{x}(b) d x \quad \text { for } \quad b \in B, t \in G,
$$

defines a contractive projection $P_{t}$, from $B$ onto $B_{t}$, where the integral is taken with respect to normalized Haar measure on $\widehat{G}$. If $e$ denotes the unit of $G$, then $P_{e}$ is in fact a positive conditional expectation onto $B_{e}$.

The collection $\mathscr{B}=\left\{B_{t}\right\}_{t \in G}$ therefore constitutes a Fell bundle over $G$. Since abelian groups are amenable we conclude, from [7, 4.7] in combination with $[7,4.2]$, that $B$ is isomorphic to both the full and the reduced crosssectional $C^{*}$-algebra of $\mathscr{B}$ ([8, VIII.17.2], [7, 2.3]).

Now suppose that, in addition to the action $\gamma$ above, we are given an action $\theta$ of $G$ on $B$ which commutes with $\gamma$, in the sense that each $\gamma_{x}$ commutes with each $\theta_{t}$. It then follows that $\theta_{s}\left(B_{t}\right) \subseteq B_{t}$ for each $t, s$ in $G$, so that $\theta$ defines an action of $G$ on the Fell bundle $\mathscr{B}$. This can in turn be fed to the 
construction described in section 2 , providing the $\theta$-deformed bundle $\mathscr{B}^{\theta}$, and its cross-sectional $C^{*}$-algebra.

Definition 4.1. Given commuting actions $\gamma$ and $\theta$, respectively of $\widehat{G}$ and $G$, on the $C^{*}$-algebra $B$, the cross-sectional $C^{*}$-algebra of $\mathscr{B}^{\theta}$ will be denoted $B_{\gamma}^{\theta}$.

Notice that, if $\theta$ is the trivial action, then $\mathscr{B}^{\theta}=\mathscr{B}$, so $B_{\gamma}^{\theta}=B$. Likewise, if $\gamma$ is trivial then $B_{t}=\{0\}$, for all $t$, except for $B_{e}$ which is all of $B$ and, again $B_{\gamma}^{\theta}=B$. However, if neither group acts trivially, then the algebraic structure of $B$ may suffer a significant transformation as it will become apparent after we discuss a few examples.

Definition 4.2. A deformation data for a $C^{*}$-algebra $B$ consists of a triple $(G, \gamma, \theta)$, where $G$ is a discrete abelian group, and $\gamma$ and $\theta$ are commuting actions, respectively of $\widehat{G}$ and $G$, on $B$. The action $\gamma$ will be called the gauge action while $\theta$ will be referred to as the deforming action.

Unless otherwise noted, the Fell bundle $\mathscr{B}=\left\{B_{t}\right\}_{t \in G}$, in the context of a deformation data $(G, \gamma, \theta)$ for a $C^{*}$-algebra $B$, refers to the spectral decomposition for the gauge action, as above.

Remark 4.3. Observe that $B_{\gamma}^{\theta}$, being the cross-sectional $C^{*}$-algebra of $\mathscr{B}^{\theta}$, contains the algebraic direct sum $\bigoplus_{t \in G} B_{t}$ as a dense *-sub-algebra. Now, the set $\bigoplus_{t \in G} B_{t}$ itself, as well as its linear structure, and the norm on each fiber, depends exclusively on the gauge action. However, its involution and multiplication operations are strongly dependent on the deforming action. Also, since the fibers of $\mathscr{B}^{\theta}$ embed isometrically into its cross-sectional $C^{*}$-algebra, we see that the norm of an element belonging to a fiber remains unaffected by the deformation. However, there is not much we can say about the norm of other elements in $\bigoplus_{t \in G} B_{t}$. Summarizing, in case we are given several deformation data sharing the same gauge action, it will be convenient to think of the deformed algebras as completions of $\bigoplus_{t \in G} B_{t}$ under different norms and with different algebraic operations.

Proposition 4.4. Let $(G, \gamma, \theta)$ be a deformation data for a $C^{*}$-algebra $B$. Suppose $B$ carries a third continuous action $\alpha$, this time of a locally compact group $H$, which commutes both with $\gamma$ and $\theta$. Then there exists a continuous action $\tilde{\alpha}$ of $H$ on $B_{\gamma}^{\theta}$ which coincides with $\alpha$ on $\bigoplus_{t \in G} B_{t}$.

Proof. Since $\alpha$ commutes with the gauge action, each spectral subspace $B_{t}$ is invariant by $\alpha_{h}$, for each $h \in H$. So $\alpha_{h}$ can be thought of as an automorphism of the Fell bundle $\mathscr{B}$. We claim it is also automorphic for the deformed structure. In fact, if $b_{t} \in B_{t}$ and $b_{s} \in B_{s}$ then

$$
\alpha_{h}\left(b_{t} \times b_{s}\right)=\alpha_{h}\left(b_{t} \theta_{t}\left(b_{s}\right)\right)=\alpha_{h}\left(b_{t}\right) \theta_{t}\left(\alpha_{h}\left(b_{s}\right)\right)=\alpha_{h}\left(b_{t}\right) \times \alpha_{h}\left(b_{s}\right),
$$


and

$$
\alpha_{h}\left(b_{t}^{\diamond}\right)=\alpha_{h}\left(\theta_{t}^{-1}\left(b_{t}^{*}\right)\right)=\theta_{t}^{-1}\left(\alpha_{h}\left(b_{t}\right)^{*}\right)=\alpha_{h}\left(b_{t}\right)^{\diamond} .
$$

Thus $\alpha_{h}$ extends to an automorphism of $B_{\gamma}^{\theta}$. The remaining verifications are left to the reader.

Notice that, in particular, one can take the action $\alpha$ above to be the gauge action itself, so one can speak of the "deformed gauge action' $\tilde{\gamma}$.

Proposition 4.5. For $t$ in $G$, the $t$-spectral subspace for the deformed gauge action on $B_{\gamma}^{\theta}$ is $B_{t}$.

Proof. Let us denote the $t$-spectral subspace for $\tilde{\gamma}$ by $\tilde{B}_{t}$. Since $\tilde{\gamma}$ coincides with $\gamma$ on $\bigoplus_{t \in G} B_{t}$, it is clear that $\tilde{\gamma}_{x}\left(b_{t}\right)=\langle x, t\rangle b_{t}$ for each $b_{t}$ in $B_{t}$. So $B_{t} \subseteq \tilde{B}_{t}$. Conversely, if $a \in \tilde{B}_{t}$, and $\varepsilon>0$, take a finite sum $\sum_{r \in G} b_{r}$ with $b_{r} \in B_{r}$, and such that $\left\|a-\sum_{r \in G} b_{r}\right\|<\varepsilon$. Considering the spectral projections

$$
\tilde{P}_{t}(b)=\int_{\widehat{G}}\langle x, t\rangle^{-1} \tilde{\gamma}_{x}(b) d x \quad \text { for } \quad b \in B_{\gamma}^{\theta}, t \in G,
$$

we have $a=\tilde{P}_{t}(a)$ while $\tilde{P}_{t}\left(\sum_{r \in G} b_{r}\right)=b_{t}$. So $\left\|a-b_{t}\right\|=\| \tilde{P}_{t}(a-$ $\left.\sum_{r \in G} b_{r}\right) \|<\varepsilon$. Therefore $a$ is in the closure of $B_{t}$ in $B_{\gamma}^{\theta}$. But, since the norm on $B_{t}$ is not affected by the deformation, $B_{t}$ is closed in $B_{\gamma}^{\theta}$, and $a \in B_{t}$.

THeOREM 4.6. Let $(G, \gamma, \theta)$ be a deformation data for a $C^{*}$-algebra $B$, and let $\alpha$ be an action of a group $H$ on $B$ which commutes both with $\gamma$ and $\theta$. Let $B^{0}$ be the fixed point sub-algebra of $B$ for $\alpha$, and let $\gamma^{0}$ and $\theta^{0}$ be the restrictions of $\gamma$ and $\theta$ to $B^{0}$, respectively. Then the deformed algebra $\left(B^{0}\right)_{\gamma^{0}}^{\theta^{0}}$ is isomorphic, to the fixed point sub-algebra of $B_{\gamma}^{\theta}$ for $\tilde{\alpha}$.

Proof. Observe that, since $\tilde{\alpha}$ and $\tilde{\gamma}$ coincide with $\alpha$ and $\gamma$, respectively, on $\bigoplus_{t \in G} B_{t}$, then they commute. This implies that the fixed point sub-algebra $A$ for $\tilde{\alpha}$ is invariant under $\tilde{\gamma}$. It follows from 4.5 , that the spectral decomposition of the restriction of $\tilde{\gamma}$ to $A$ is $\bigoplus_{t \in G} B_{t} \cap A$. Now, since $\alpha$ and $\tilde{\alpha}$ agree on each $B_{t}$,

$$
\begin{gathered}
B_{t} \cap A=\left\{b \in B_{t}: \alpha_{h}(b)=b \text { for all } h \in H\right\} \\
=\left\{b \in B: \alpha_{h}(b)=b \text { for all } h \in H \text { and } \gamma_{x}(b)=\langle x, t\rangle b \text { for all } x \in \widehat{G}\right\} \\
=\left\{b \in B^{0}: \gamma_{x}(b)=\langle x, t\rangle b, \text { for all } x \in \widehat{G}\right\}=B_{t}^{0},
\end{gathered}
$$

where we have denoted by $B_{t}^{0}$ the $t$-spectral subspace of $B^{0}$ under $\gamma^{0}$. It is now easy to see that the Fell bundle structure arising from the grading $\left\{B_{t} \cap A\right\}_{t \in G}$ of $A$, and that of the grading of the deformed algebra $\left(B^{0}\right)_{\gamma^{0}}^{\theta^{0}}$ are isomorphic. The result then follows from [7, 4.2]. 


\section{The derivative of the deformed product}

Let $B$ be a $C^{*}$-algebra carrying a strongly continuous action $\phi$ of $\mathrm{R}^{2 d}$. For each $j=1, \ldots, 2 d$, define the differential operator $\partial_{u_{j}}$ on $B$ by

$$
\partial_{u_{j}}(f)=\left.\frac{d}{d \lambda}\left(\phi_{(0, \ldots, \lambda, \ldots, 0)}(f)\right)\right|_{\lambda=0}, \quad \text { for } \quad f \in B,
$$

where the $\lambda$ in $(0, \ldots, \lambda, \ldots, 0)$ appears in the $j^{\text {th }}$ position. Of course $\partial_{u_{j}}(f)$ is only defined when $f$ is sufficiently smooth. In particular this is the case for the $\phi$-smooth elements, that is, those elements $f \in B$ such that $u \in \mathrm{R}^{2 d} \longmapsto$ $\phi_{u}(f) \in B$ is an infinitely differentiable Banach space valued function. It is well known that these elements form a dense subset of $B$ (see, e.g, [3, 2.2.1]).

In what follows we shall adopt the coordinate system $\left(x_{1}, \ldots, x_{d}, y_{1}, \ldots, y_{d}\right)$ on $\mathrm{R}^{2 d}$ and hence we shall speak of the differential operators $\partial_{x_{j}}$ and $\partial_{y_{j}}$, for $j=1, \ldots, d$.

In [17] (see also [18]) Rieffel showed how to construct a strict deformation quantization of $B$ "in the direction" of the Poisson bracket $\{\cdot, \cdot\}$ defined by

$$
\{f, g\}=\sum_{j=1}^{d} \partial_{x_{j}}(f) \partial_{y_{j}}(g)-\partial_{y_{j}}(f) \partial_{x_{j}}(g),
$$

when $B$ is the algebra of continuous functions on a smooth manifold. Rieffel deals, in fact, with a more general situation, where the Poisson bracket involves the choice of a skew-symmetric matrix $J$.

In order to describe a connection between Rieffel's theory and ours, we next compute the derivative of the deformed product on $B$, arising from a certain deformation data associated to $\phi$.

Let $\gamma$ be the action of $\mathrm{R}^{d}$ given by the restriction of $\phi$ to its first $d$ variables, that is

$$
\gamma_{\left(x_{1}, \ldots, x_{d}\right)}=\phi_{\left(x_{1}, \ldots, x_{d}, 0, \ldots, 0\right)}, \quad \text { for }\left(x_{1}, \ldots, x_{d}\right) \in \mathrm{R}^{d} .
$$

The technical complications will be kept to a minimum by assuming that $\phi$ is periodic in the first $d$ variables, so that $\gamma$ defines an action of the torus $\mathrm{T}^{d}$ on $B$, which we still denote by $\gamma$.

On the other hand, consider the action $\theta$ of $\mathrm{R}^{d}$ on $B$ defined by

$$
\theta_{\left(y_{1}, \ldots, y_{d}\right)}=\phi_{\left(0, \ldots, 0, y_{1}, \ldots, y_{d}\right)}, \quad \text { for }\left(y_{1}, \ldots, y_{d}\right) \in \mathrm{R}^{d} .
$$

If $\hbar$ is a real number, we will let the action $\theta^{\hbar}$ of $Z^{d}$ on $B$ be defined by

$$
\theta_{\left(n_{1}, \ldots, n_{d}\right)}^{\hbar}=\theta_{\left(\hbar n_{1}, \ldots, \hbar n_{d}\right)}, \quad \text { for }\left(n_{1}, \ldots, n_{d}\right) \in Z^{d} .
$$


Since $\gamma$ and $\theta^{\hbar}$ commute, the triple $\left(Z^{d}, \gamma, \theta^{\hbar}\right)$ is a deformation data for $B$.

Let $\mathscr{B}=\left\{B_{t}\right\}_{t \in G}$ be the Fell bundle arising from the spectral decomposition of $\gamma$. We denote the operations on the deformed bundle $\mathscr{B}^{\theta^{\hbar}}$ by $\times_{\hbar}$ and ${ }^{\diamond_{h}}$, and the deformed algebra $B_{\gamma}^{\theta^{\hbar}}$ by $B^{(\hbar)}$.

Proposition 5.1. If $f$ is $\phi$-smooth then $P_{t}(f)$ is also $\phi$-smooth for all $t$ in $\mathrm{Z}^{d}$. In addition, for $j=1, \ldots, 2 d$, we have $\partial_{u_{j}}\left(P_{t}(f)\right)=P_{t}\left(\partial_{u_{j}}(f)\right)$, and therefore each $B_{t}$ is invariant under $\partial_{u_{j}}$.

Proof. For $u \in \mathrm{R}^{2 d}$ we have

$$
\phi_{u}\left(P_{t}(f)\right)=\phi_{u}\left(\int_{\mathrm{T}^{d}}\langle x, t\rangle^{-1} \gamma_{x}(f) d x\right)=\int_{\mathrm{T}^{d}}\langle x, t\rangle^{-1} \gamma_{x}\left(\phi_{u}(f)\right) d x,
$$

which is therefore smooth as a function of $u$. This shows that $P_{t}(f)$ is $\phi$ smooth. We have

$$
\begin{aligned}
\partial_{u_{j}}\left(P_{t}(f)\right) & =\left.\frac{d}{d \lambda}\left(\phi_{(0, \ldots, \lambda, \ldots, 0)} P_{t}(f)\right)\right|_{\lambda=0} \\
& =\left.\int_{\top^{d}} \frac{d}{d \lambda}\left(\phi_{(0, \ldots, \lambda, \ldots, 0)}\left(\langle x, t\rangle^{-1} \gamma_{x}(f)\right)\right)\right|_{\lambda=0} d x \\
& =\int_{\top^{d}}\langle x, t\rangle^{-1} \gamma_{x}\left(\partial_{u_{j}}(f)\right) d x=P_{t}\left(\partial_{u_{j}}(f)\right) .
\end{aligned}
$$

Lemma 5.2. Let $t=\left(t_{1}, \ldots, t_{d}\right)$ and $s=\left(s_{1}, \ldots, s_{d}\right)$ be in $\mathrm{Z}^{d}$ and take $f \in B_{t}$ and $g \in B_{s}$. Suppose that $g$ is smooth for $\theta$. Then, for all real numbers $\hbar$

$$
\left\|\frac{f \times_{\hbar} g-f g}{\hbar}-\frac{1}{2 \pi i} \sum_{j=1}^{d} \partial_{x_{j}}(f) \partial_{y_{j}}(g)\right\| \leq|\hbar|\|f\|\left\|\sum_{j, k=1}^{d} t_{j} t_{k} \partial_{y_{j}}\left(\partial_{y_{k}}(g)\right)\right\| .
$$

Proof. Notice that the term whose norm appears in the left hand side above lies in $B_{t+s}$, which is isometrically embedded into each $B^{(\hbar)}$, so its norm is unambiguously defined. We have

$$
f \times_{\hbar} g-f g=f \theta_{t}^{\hbar}(g)-f g .
$$

Now, consider the $C^{\infty}$ map $F: \mathrm{R} \rightarrow B$ given by

$$
F(\hbar):=\theta_{t}^{\hbar}(g)=\phi_{\left(0, \ldots, 0, \hbar t_{1}, \ldots, h t_{d}\right)}(g) .
$$

Its first two derivatives are given by

$$
F^{\prime}(\hbar)=\phi_{\left(0, \ldots, 0, \hbar t_{1}, \ldots, \hbar t_{d}\right)}\left(\sum_{j=1}^{d} t_{j} \partial_{y_{j}}(g)\right),
$$


and

$$
F^{\prime \prime}(\hbar)=\phi_{\left(0, \ldots, 0, \hbar t_{1}, \ldots, \hbar t_{d}\right)}\left(\sum_{j, k=1}^{d} t_{j} t_{k} \partial_{y_{j}}\left(\partial_{y_{k}}(g)\right)\right),
$$

for all $\hbar$ in R. The first order Taylor expansion for $F$ reads

$$
F(\hbar)=F(0)+\hbar F^{\prime}(0)+\int_{0}^{\hbar}(\hbar-\lambda) F^{\prime \prime}(\lambda) d \lambda
$$

from where we conclude that

$$
\left\|\frac{F(\hbar)-F(0)}{\hbar}-F^{\prime}(0)\right\| \leq|\hbar| \sup _{\lambda \in I}\left\|F^{\prime \prime}(\lambda)\right\|,
$$

where $I$ is either $[0, \hbar]$ or $[\hbar, 0]$, depending on the sign of $\hbar$. In terms of $g$, we get

$$
\left\|\frac{\theta_{t}^{\hbar}(g)-g}{\hbar}-\sum_{j=1}^{d} t_{j} \partial_{y_{j}}(g)\right\| \leq|\hbar|\left\|\sum_{j, k=1}^{d} t_{j} t_{k} \partial_{y_{j}}\left(\partial_{y_{k}}(g)\right)\right\| .
$$

Using the first equation obtained in this proof gives

$$
\left\|\frac{f \times_{\hbar} g-f g}{\hbar}-\sum_{j=1}^{d} t_{j} f \partial_{y_{j}}(g)\right\| \leq|\hbar|\|f\|\left\|\sum_{j, k=1}^{d} t_{j} t_{k} \partial_{y_{j}}\left(\partial_{y_{k}}(g)\right)\right\| .
$$

On the other hand, recall that $f$ is in the $t$-spectral subspace of the gauge action. This means that, for $x=\left(x_{1}, \ldots, x_{d}\right) \in \mathrm{R}^{d}$, we have that $\gamma_{x}(f)=\langle x, t\rangle f$, or

$$
\gamma_{x}(f)=\mathrm{e}^{2 \pi i x_{1} t_{1}} \ldots \mathrm{e}^{2 \pi i x_{d} t_{d}} f .
$$

If follows that $\partial_{x_{j}}(f)=2 \pi i t_{j} f$, and hence that $t_{j} f=(2 \pi i)^{-1} \partial_{x_{j}}(f)$, which, when plugged into the last inequality above, leads to the conclusion.

The purpose of this Lemma is to allow us to compute the derivative of $f \times_{\hbar} g$, with respect to $\hbar$. However, the expression $f \times_{\hbar} g$, applies only for $f$ and $g$ belonging, each, to a spectral subspace of the gauge action. The question we want to address is:

Question 5.3. What is the biggest subset of $B$ that can be mapped, in a natural way, into each deformed algebra $B^{(\hbar)}$ ?

The remark made in 4.3 provides $\bigoplus_{t \in Z^{d}} B_{t}$ as a partial answer. Now, since $B^{(\hbar)}$ contains a copy of the $L_{1}$ cross-sectional algebra $L_{1}(\mathscr{B})$ which, again by 4.3 , does not depend on $\hbar$, as far as its normed linear space structure is concerned, $L_{1}(\mathscr{B})$ is a better answer to our question. 
We do not claim, however, that this is the best possible answer. In fact, the word natural in 5.3 lacks a precise meaning, as it stands. The correct way to rephrase 5.3 could possibly be:

Question 5.4. For each $\hbar$, let $\iota^{\hbar}: L_{1}(\mathscr{B}) \rightarrow B^{(\hbar)}$ be the natural inclusion, viewed as a densely defined linear map on $B$. Is $\iota^{\hbar}$ closable? That is, is the closure of its graph, the graph of a well defined linear map? If so, how to characterize the domain $D^{\hbar}$ of this map? Is there any relationship between the $D^{\hbar}$ for different $\hbar$ ? What is the intersection of the $D^{\hbar}$ as $\hbar$ ranges in R?

An advantage of $L_{1}(\mathscr{B})$ is that it includes the smooth elements for the gauge action: it is a well known fact that, for such an element $f$, one has that $f=\sum_{t \in Z^{d}} P_{t}(f)$, where the series is absolutely convergent.

TheOREm 5.5. Let $f, g \in B$ be $\phi$-smooth elements. Then

$$
\lim _{\hbar \rightarrow 0}\left\|\frac{f \times_{\hbar} g-f g}{\hbar}-\frac{1}{2 \pi i} \sum_{j=1}^{d} \partial_{x_{j}}(f) \partial_{y_{j}}(g)\right\|_{\hbar}=0,
$$

where $\|\cdot\|_{\hbar}$ refers to the norm of the deformed algebra $B^{(\hbar)}$.

Proof. First notice that the terms appearing between the double bars above can be viewed as elements of $B^{(\hbar)}$. This is because the smooth elements $f, g$, $f g$, and $\partial_{x_{j}}(f) \partial_{y_{j}}(g)$, may be seen as elements of $L_{1}(\mathscr{B})$, which, in turn, may be interpreted as a subset of $B^{(\hbar)}$, according to the comment above.

Write $f=\sum_{t \in Z^{d}} P_{t}(f)$ and $g=\sum_{t \in Z^{d}} P_{t}(g)$. For each $j=1, \ldots, 2 d$ we have that $\partial_{u_{j}}(f)$ is also smooth, hence it "Fourier series" converges:

$$
\partial_{u_{j}}(f)=\sum_{t \in Z^{d}} P_{t}\left(\partial_{u_{j}}(f)\right)=\sum_{t \in Z^{d}} \partial_{u_{j}}\left(P_{t}(f)\right),
$$

and similarly for $g$. So,

$$
\sum_{j=1}^{d} \partial_{x_{j}}(f) \partial_{y_{j}}(g)=\sum_{t, s \in Z^{d}} \sum_{j=1}^{d} \partial_{x_{j}}\left(P_{t}(f)\right) \partial_{y_{j}}\left(P_{s}(g)\right) .
$$

Also

$$
\frac{f \times_{\hbar} g-f g}{\hbar}=\sum_{t, s \in Z^{d}} \frac{P_{t}(f) \times_{\hbar} P_{s}(g)-P_{t}(f) P_{s}(g)}{\hbar}
$$

Using 5.2, it follows that

$$
\left\|\frac{f \times_{\hbar} g-f g}{\hbar}-\frac{1}{2 \pi i} \sum_{j=1}^{d} \partial_{x_{j}}(f) \partial_{y_{j}}(g)\right\|_{\hbar}
$$




$$
\begin{gathered}
\leq \sum_{t, s \in Z^{d}}\left\|\frac{P_{t}(f) \times_{\hbar} P_{s}(g)-P_{t}(f) P_{s}(g)}{\hbar}-\frac{1}{2 \pi i} \sum_{j=1}^{d} \partial_{x_{j}}\left(P_{t}(f)\right) \partial_{y_{j}}\left(P_{s}(g)\right)\right\|_{\hbar} \\
\leq|\hbar| \sum_{t, s \in Z^{d}}\left\|P_{t}(f)\right\|\left\|\sum_{j, k=1}^{d} t_{j} t_{k} \partial_{y_{j}}\left(\partial_{y_{k}}\left(P_{s}(g)\right)\right)\right\| \\
\leq|\hbar| \sum_{j, k=1}^{d}\left(\sum_{t \in Z^{d}}\left|t_{j} t_{k}\right|\left\|P_{t}(f)\right\|\right)\left(\sum_{s \in Z^{d}}\left\|P_{s}\left(\partial_{y_{j}}\left(\partial_{y_{k}}(g)\right)\right)\right\|\right) .
\end{gathered}
$$

By our hypothesis, these infinite series converge, and hence the whole thing tends to zero as $\hbar \rightarrow 0$.

REMARK 5.6. If one is interested in determining the exact class of differentiability needed for the above result to hold, a quick look at the last displayed expression, in the proof above, gives the answer. That is, $f$ should be supposed to be of class $C^{2 d+2}$ for $\gamma$, and the second order differential of $g$ with respect to $\theta$ should be of class $C^{2 d}$ for $\gamma$. These conditions imply the convergence of these infinite series, and hence the conclusion.

Our next result shows that the derivative of the commutator, for the deformed product, is given by the Poisson bracket described at the beginning of this section. Its proof is an immediate consequence of 5.5.

Corollary 5.7. Let $f, g \in B$ be smooth elements for $\phi$. Then

$$
\lim _{\hbar \rightarrow 0}\left\|\frac{f \times_{\hbar} g-g \times_{\hbar} f-[f, g]}{\hbar}-\frac{1}{2 \pi i}\{f, g\}\right\|_{\hbar}=0,
$$

where $[\cdot, \cdot \cdot]$ is the commutator for the original multiplication on $B$, and $\{\cdot, \cdot\}$ is the Poisson bracket defined near the beginning of this section.

Since the family $\left\{\theta^{\hbar}\right\}_{\hbar \in R}$ is obviously continuous in the sense of section 3, we get, by 3.5 , a continuous field of $C^{*}$-algebras $\left\{B^{(\hbar)}\right\}_{\hbar \in \mathrm{R}}$, and hence a strict deformation quantization in the sense of Rieffel [16, Definition 1.1], with the modification, required in the noncommutative situation, corresponding to the introduction of the term $[f, g]$ in the statement of 5.7. We have thus shown:

Corollary 5.8. The family $\left\{B^{(\hbar)}\right\}_{\hbar \in \mathrm{R}}$ gives a strict deformation quantization for $B$, in the direction of the Poisson bracket defined above.

\section{Example: Non commutative 3-spheres}

In [10] Matsumoto defined a family of $C^{*}$-algebras, denoted $S_{\vartheta}^{3}$, depending on a real parameter $\vartheta$. This family is a deformation of the commutative $C^{*}$ algebra $C\left(S^{3}\right)$ of all continuous complex valued functions on the 3 -sphere $S^{3}$, 
because, when $\vartheta=0, S_{\vartheta}^{3}$ is isomorphic to $C\left(S^{3}\right)$. The purpose of the present section is to show that $S_{\vartheta}^{3}$ can be constructed from a certain deformation data for the algebra $C\left(S^{3}\right)$. Recall from [10] that $S_{\vartheta}^{3}$ may be defined as the universal $C^{*}$-algebra given by generators and relations as follows: for generators take symbols $S$ and $T$ and for relations consider

M-1) $S^{*} S=S S^{*}, T^{*} T=T T^{*}$,

M-2) $\|S\| \leq 1,\|T\| \leq 1$,

M-3) $\left(1-T^{*} T\right)\left(1-S^{*} S\right)=0$, and

M-4) $T S=\mathrm{e}^{2 \pi i \vartheta} S T$.

An alternative description of $S_{\vartheta}^{3}$ is given by [10, 8.1]. It says that $S_{\vartheta}^{3}$ is also the universal $C^{*}$-algebra on the generators $B$ and $C$ satisfying

M-1') $B^{*} B=B B^{*}, C^{*} C=C C^{*}$,

M-2') $B^{*} B+C^{*} C=1$, and

M-3') $C B=\mathrm{e}^{2 \pi i \vartheta} B C$.

The relationship between these presentations is given by the formulas

$$
B=S\left(S^{*} S+T^{*} T\right)^{-\frac{1}{2}}, \quad C=T\left(S^{*} S+T^{*} T\right)^{-\frac{1}{2}} .
$$

Define an action $\gamma$ of $S^{1}$ on $S^{3}$ by $\gamma_{\lambda}(z, w)=(\lambda z, \lambda w)$, where $z, w, \lambda \in \mathrm{C}$ satisfy $|z|^{2}+|w|^{2}=1$ and $|\lambda|=1$.

Also, fixing a real number $\vartheta$, define an action $\theta$ of $Z$ on $S^{3}$ by $\theta_{n}(z, w)=$ $\left(\mathrm{e}^{2 \pi i n \vartheta} z, w\right)$, for $(z, w) \in S^{3}, n \in Z$. These give actions of $S^{1}$ and $Z$ on $C\left(S^{3}\right)$ by letting

$$
\left.\gamma_{\lambda}(f)\right|_{(z, w)}=f(\lambda z, \lambda w), \quad \text { and }\left.\quad \theta_{n}(f)\right|_{(z, w)}=f\left(\mathrm{e}^{2 \pi i n \vartheta} z, w\right)
$$

for $f \in C\left(S^{3}\right),(z, w) \in S^{3}, \lambda \in S^{1}$ and $n \in Z$. Noting that $\gamma$ and $\theta$ commute with each other, we see that we are facing a deformation data $(Z, \gamma, \theta)$ for the algebra $C\left(S^{3}\right)$.

THeOREM 6.1. The deformed algebra $C\left(S^{3}\right)_{\gamma}^{\theta}$ is isomorphic to Matsumoto's algebra $S_{\vartheta}^{3}$.

Proof. Let $Z, W \in C\left(S^{3}\right)$ be the functions defined by $Z(z, w)=z$, and $W(z, w)=w$, for $(z, w) \in S^{3}$. Since $\gamma_{\lambda}(Z)=\lambda Z$ and $\gamma_{\lambda}(W)=\lambda W$, we have that both $Z$ and $W$ belong to the first spectral subspace for $\gamma$. Then, regarding the deformed product, we have

$$
Z \times W=Z \theta_{1}(W)=Z W \text { and } W \times Z=W \theta_{1}(Z)=\mathrm{e}^{2 \pi i \vartheta} W Z,
$$


so that $W \times Z=\mathrm{e}^{2 \pi i \vartheta} Z \times W$. This says that $Z$ and $W$ satisfy (M-3'). It is easy to check that they also satisfy (M-1') and (M-2') with respect to the deformed product an involution. So, by the universal property, there exists a $C^{*}$-algebra homomorphism

$$
\psi: S_{\vartheta}^{3} \rightarrow C\left(S^{3}\right)_{\gamma}^{\theta}
$$

such that $\psi(B)=Z$ and $\psi(C)=W$, which we claim to be an isomorphism.

To show that $\psi$ is surjective observe that, since $Z$ and $W$ belong to the image of $\psi$, we just have to show that $Z$ and $W$ generate $C\left(S^{3}\right)_{\gamma}^{\theta}$. We first show that the $n$-spectral subspace for the action $\gamma$ on $C\left(S^{3}\right)$ is linearly spanned by the set

$$
\left\{Z^{i} Z^{* j} W^{k} W^{* l}: i, j, k, l \in \mathbf{N}, i-j+k-l=n\right\} .
$$

In fact, any $f \in C\left(S^{3}\right)$ may be arbitrarily approximated by a linear combination of terms of the form $Z^{i} Z^{* j} W^{k} W^{* l}$. Now, if $f$ belongs to the $n$-spectral subspace, then $f=P_{n}(f)$, where $P_{n}$ is the corresponding spectral projection. On the other hand, if $P_{n}$ is applied to the linear combination just mentioned, all terms will vanish except for those for which $i-j+k-l=n$.

The fact that $Z$ and $W$ are also eigenvalues for $\theta$ implies that

$$
Z^{i} \times Z^{* j} \times W^{k} \times W^{* l}=\mu Z^{i} Z^{* j} W^{k} W^{* l},
$$

for some complex number $\mu$ of modulus one. Therefore one concludes that each spectral subspace for the deformed gauge action is contained in the subalgebra of $C\left(S^{3}\right)_{\gamma}^{\theta}$ generated by $Z$ and $W$. This shows that $Z$ and $W$ generate $C\left(S^{3}\right)_{\gamma}^{\theta}$ and hence that $\psi$ is surjective.

We next show that $\psi$ is injective. Consider the circle action on $S_{\vartheta}^{3}$ specified, on the generators, by

$$
\alpha_{\lambda}(B)=\lambda B \quad \text { and } \quad \alpha_{\lambda}(C)=\lambda C,
$$

for $\lambda \in S^{1}$. The homomorphism $\psi$ is clearly equivariant for the action just defined on $S_{\vartheta}^{3}$ and the deformed gauge action $\tilde{\gamma}$ on $C\left(S^{3}\right)_{\gamma}^{\theta}$. By using [6, 2.9], it is now enough to verify that $\psi$ is injective on the fixed point sub-algebra of $S_{\vartheta}^{3}$ for $\alpha$. Let us denote that sub-algebra by $F$.

Recall that Matsumoto [11, Theorem 6] has shown that $F$ is isomorphic to the commutative $C^{*}$-algebra of functions on the two-sphere $S^{2}$. More precisely, $F$ turns out to be generated by the elements $M$ and $H$ of $S_{\vartheta}^{3}$ given by $H=C^{*} C$ and $M=C B^{*}$. It is easy to see that these operators satisfy the relations

i) $H^{*}=H$,

ii) $M^{*} M=M M^{*}$, 
iii) $M H=H M$, and

iv) $M^{*} M+H^{2}=H$.

Matsumoto has, in fact, shown that $F$ is the universal $C^{*}$-algebra on generators $H$ and $M$ satisfying the above relations.

Now, the images of $H$ and $M$ under $\psi$ are

$$
\psi(H)=W^{\diamond} \times W=W^{*} W \text { and } \psi(M)=W \times Z^{\diamond}=W Z^{*},
$$

both of which lie in the fixed point sub-algebra, say $B_{0}$, for the deformed gauge action on $C\left(S^{3}\right)_{\gamma}^{\theta}$. The crucial point is that this algebra is not affected by the deformation, so $B_{0}$ is just the algebra of continuous functions on the quotient space $S^{3} / S^{1}$, which is homeomorphic to $S^{2}$.

An explicit homeomorphism between $S^{3} / S^{1}$ and $S^{2}$ may be given by mapping the quotient class of $(z, w) \in S^{3}$ to the pair $(h, m) \in \mathrm{R} \times \mathrm{C}$, defined by $(h, m)=(w \bar{w}, w \bar{z})$. It is elementary to check that $(h, m)$ satisfies the equation

$$
|m|^{2}+h^{2}=h
$$

which is precisely the equation defining the sphere of radius $\frac{1}{2}$ centered at $\left(\frac{1}{2}, 0+i 0\right)$ in $\mathrm{R} \times \mathrm{C}$. The map

$$
\overparen{(z, w)} \longmapsto(w \bar{w}, w \bar{z})
$$

can now be shown to provide a homeomorphism from $S^{3} / S^{1}$ onto the above mentioned model for the 2-sphere.

When a compact subset $K$ of $\mathrm{R} \times \mathrm{C}$ is defined via a system of equations, such as the sphere above, it is well-known that $C(K)$ is the universal $C^{*}$-algebra generated by symbols $h$ and $m$, subject to the conditions

i) $h^{*}=h$,

ii) $m^{*} m=m m^{*}$,

iii) $m h=h m$,

to which one should add the equations used to define $K$. This implies that $B_{0}$ is the universal $C^{*}$-algebra generated by a pair of elements (namely $h=W^{*} W$ and $\left.m=W Z^{*}\right)$ subject to the same relations as the ones defining $F$, that is 6.2 .

Therefore one sees that $\psi$ is an isomorphism between $F$ and $B_{0}$, hence injective. By $[6,2.9]$, it follows that $\psi$ is injective everywhere and thus it is an isomorphism. 
In order to discuss the derivative of the deformation of $S^{3}$ just described, let $D_{1}$ and $D_{2}$ denote the differential operators defined by

$$
D_{1} f(z, w)=\left.\frac{d}{d \lambda}\left(f\left(\mathrm{e}^{2 \pi i \lambda} z, w\right)\right)\right|_{\lambda=0},
$$

and

$$
D_{2} f(z, w)=\left.\frac{d}{d \lambda}\left(f\left(z, \mathrm{e}^{2 \pi i \lambda} w\right)\right)\right|_{\lambda=0},
$$

for $(z, w) \in S^{3}$ and $f \in C^{\infty}\left(S^{3}\right)$.

THEOREM 6.3. If $f$ and $g$ are in $C^{\infty}\left(S^{3}\right)$ then

$$
\lim _{\vartheta \rightarrow 0}\left\|\frac{f \times_{\vartheta} g-g \times \times_{\vartheta} f}{\vartheta}-\frac{1}{2 \pi i}\left(D_{2}(f) D_{1}(g)-D_{1}(f) D_{2}(g)\right)\right\|_{\vartheta}=0,
$$

where $\times_{\vartheta}$ and $\|\cdot\|_{\vartheta}$ refer to the deformed multiplication and norm of $S_{\vartheta}^{3}$. Therefore, the family $\left\{S_{\vartheta}^{3}\right\}_{\vartheta \in \mathrm{R}}$ gives a strict deformation quantization for $C\left(S^{3}\right)$, in the direction of the Poisson bracket $D_{2} \wedge D_{1}$.

Proof. Let $\phi$ be the action of $\mathrm{R}^{2}$ on $S^{3}$ defined by

$$
\phi_{(x, y)}(z, w)=\left(\mathrm{e}^{2 \pi i(x+y)} z, \mathrm{e}^{2 \pi i x} w\right), \quad \text { for }(x, y) \in \mathrm{R}^{2},(z, w) \in S^{3} .
$$

As in section 5 we may use $\phi$ to obtain the deformation data $\left(Z, \gamma, \theta^{\hbar}\right)$. However, one can easily see that this is precisely the deformation data used earlier in this section for $\hbar=\vartheta$. So 5.7 applies to the deformation $S_{\vartheta}^{3}$. Notice that, since $\phi$ is a smooth action of $\mathrm{R}^{2}$ on the compact manifold $S^{3}$, then any smooth function on $S^{3}$ will be $\phi$-smooth. Also, for $f$ in $C^{\infty}\left(S^{3}\right)$ we have, using the notation of section 5 ,

$$
\left.\partial_{x}(f)\right|_{(z, w)}=\left.\frac{d}{d \lambda}\left(f\left(\mathrm{e}^{2 \pi i \lambda} z, \mathrm{e}^{2 \pi i \lambda} w\right)\right)\right|_{\lambda=0}=D_{1} f(z, w)+D_{2} f(z, w),
$$

and

$$
\left.\partial_{y}(f)\right|_{(z, w)}=\left.\frac{d}{d \lambda}\left(f\left(\mathrm{e}^{2 \pi i \lambda} z, w\right)\right)\right|_{\lambda=0}=D_{1} f(z, w),
$$

that is, $\partial_{x}=D_{1}+D_{2}$ while $\partial_{y}=D_{1}$. The Poisson bracket appearing in 5.7 then becomes

$$
\begin{aligned}
\partial_{x}(f) \partial_{y}(g)-\partial_{y}(f) \partial_{x}(g)= & \left(D_{1}(f)+D_{2}(f)\right) D_{1}(g) \\
& -D_{1}(f)\left(D_{1}(g)+D_{2}(g)\right) \\
= & D_{2}(f) D_{1}(g)-D_{1}(f) D_{2}(g),
\end{aligned}
$$

concluding the proof. 


\section{Example: Non commutative Lens spaces}

Matsumoto and Tomiyama [12], building on [10], have introduced non-commutative versions of the classical lens spaces. This section is dedicated to proving that these can be described by using our method of deformation.

Recall that for nonzero co-prime integers $p$ and $q$, with $p \neq 0$, the lens space $L(p, q)$ is the quotient of the three-sphere $S^{3}$ by the action of the finite cyclic group $Z_{p}$ generated by the diffeomorphism

$$
\tau(z, w)=\left(\rho z, \rho^{q} w\right), \quad \text { for }(z, w) \in S^{3},
$$

where $\rho=\mathrm{e}^{2 \pi i / p}$.

Observe that, if one induces $\tau$ to an automorphism of $C\left(S^{3}\right)$ by the formula

$$
\left.\tau(f)\right|_{(z, w)}=f\left(\rho z, \rho^{q} w\right), \quad \text { for } f \in C\left(S^{3}\right), \quad(z, w) \in S^{3},
$$

then we have $\tau(Z)=\rho Z$ and $\tau(W)=\rho^{q} W$, where $Z$ and $W$ are the coordinate functions on $S^{3}$ (defined in the proof of 6.1). Since $Z$ and $W$ generate $C\left(S^{3}\right)$, these equations actually define $\tau$. In addition one sees that the fixed point subalgebra of $C\left(S^{3}\right)$ for $\tau$ coincides with the algebra of continuous functions on the quotient $S^{3} / \mathrm{Z}_{p}=L(p, q)$.

Let $\vartheta$ be a real number, fixed throughout. Consider the automorphism $\sigma$ of $S_{\vartheta}^{3}$ given by $\sigma(B)=\rho B$ and $\sigma(C)=\rho^{q} C$, where $B$ and $C$ are as in the previous section. Among many other characterizations, the non-commutative lens space $L_{\vartheta}(p, q)$ is defined in [12] to be the fixed point sub-algebra of $S_{\vartheta}^{3}$ under the automorphism $\sigma$.

Regarding the deformation data $(\mathrm{Z}, \gamma, \theta)$ for the algebra $C\left(S^{3}\right)$, defined shortly before 6.1, in terms of a given value for the parameter $\vartheta$, observe that $\gamma$ and $\theta$ commute with $\tau$ and hence both $\gamma$ and $\theta$ leave invariant the fixed point sub-algebra for $\tau$, which we have seen to be a model for $C(L(p, q))$. We still denote by $\gamma$ and $\theta$ the corresponding restrictions of these to $C(L(p, q))$. So, this gives a deformation data for $C(L(p, q))$ and we may then form the deformed algebra $C(L(p, q))_{\gamma}^{\theta}$.

THEOREM 7.1. For each real number $\vartheta$ and co-prime integers $p$ and $q$, with $p \neq 0$, the $C^{*}$-algebras $C(L(p, q))_{\gamma}^{\theta}$ and $L_{\vartheta}(p, q)$ are isomorphic.

Proof. We shall derive this from 4.6. In fact, let the algebra $B$, mentioned in the statement of 4.6 be $C\left(S^{3}\right)$ with the deformation data $(Z, \gamma, \theta)$ referred to above. Then, as we have seen in 6.1, $C\left(S^{3}\right)_{\gamma}^{\theta}$ is isomorphic to $S_{\vartheta}^{3}$. Still referring to the statement of 4.6, let $H=Z_{p}$, which acts on $B$ via $\tau$. The extension of $\tau$ to $C\left(S^{3}\right)_{\gamma}^{\theta}$, provided by 4.4 , coincides with $\tau$ on the algebraic direct sum 
of the spectral subspaces for the deformed gauge action and hence satisfies $\tilde{\tau}(Z)=\rho Z \quad$ and $\quad \tilde{\tau}(W)=\rho^{q} W$.

But, since the isomorphism between $C\left(S^{3}\right)_{\gamma}^{\theta}$ and $S_{\vartheta}^{3}$ maps $Z$ and $W$ to $B$ and $C$, respectively, we see that $\tilde{\tau}$ and $\sigma$ correspond to each other under this isomorphism. In particular, the fixed point sub-algebra for $\sigma$, that is, $L_{\vartheta}(p, q)$, is isomorphic to the fixed point sub-algebra of $C\left(S^{3}\right)_{\gamma}^{\theta}$ under $\tilde{\tau}$, which, by 4.6, is isomorphic to the deformed algebra $C(L(p, q))_{\gamma}^{\theta}$.

Observe that $\tau$ commutes with the action $\phi$ referred to in the proof of 6.3. Therefore the operators $D_{1}$ and $D_{2}$ leave $C(L(p, q))$ invariant, when this is viewed as a subset of $C\left(S^{3}\right)$.

THEOREM 7.2. If $f$ and $g$ are in $C^{\infty}(L(p, q))$ then

$$
\lim _{\vartheta \rightarrow 0}\left\|\frac{f \times_{\vartheta} g-g \times \times_{\vartheta} f}{\vartheta}-\frac{1}{2 \pi i}\left(D_{2}(f) D_{1}(g)-D_{1}(f) D_{2}(g)\right)\right\|_{\vartheta}=0,
$$

where $\times_{\vartheta}$ and $\|\cdot\|_{\vartheta}$ refer to the deformed multiplication and norm of $L_{\vartheta}(p, q)$. Therefore, the family $\left\{L_{\vartheta}(p, q)\right\}_{\vartheta \in \mathrm{R}}$ gives a strict deformation quantization for $C(L(p, q))$, in the direction of the Poisson bracket $D_{2} \wedge D_{1}$.

Proof. This is, in view of the comment above, a direct application of 6.3 for $f$ and $g$ in $C(L(p, q))$.

\section{Example: Non commutative Heisenberg manifolds}

For each positive integer $c$, the Heisenberg manifold $M^{c}$ consists of the quotient $H / G^{c}$, where $H$ is the Heisenberg group

$$
H=\left\{\left(\begin{array}{ccc}
1 & y & z \\
0 & 1 & x \\
0 & 0 & 1
\end{array}\right): x, y, z \in \mathrm{R}\right\},
$$

viewed as a subgroup of $S L_{3}(\mathrm{R})$, and $G^{c}$ is the discrete subgroup obtained when $x, y$ and $c z$ are required to be integers. In what follows we identify $H$ with $R^{3}$ via

$$
(x, y, z) \leftrightarrow\left(\begin{array}{ccc}
1 & y & z \\
0 & 1 & x \\
0 & 0 & 1
\end{array}\right)
$$

Thus, the multiplication in $H$ becomes

$$
(x, y, z)(m, n, p)=(x+m, y+n, z+p+y m) .
$$

So $M^{c}$ can be described as the quotient of the Euclidean space $\mathrm{R}^{3}$ by the right action of $G^{c}$ given by 8 . 
In [16] Rieffel introduced a continuous field of $C^{*}$-algebras, denoted $D_{\mu, \nu}^{c}$, where $\mu$ and $v$ are real parameters, such that $D_{\mu, v}^{c}$ is isomorphic to $C\left(M^{c}\right)$ when $\mu=v=0$.

Recall, from [1] and [2], that $D_{\mu, v}^{c}$ is isomorphic to the crossed product of $C\left(\mathrm{~T}^{2}\right)$, the algebra of continuous functions on the two-torus, by a Hilbert bimodule. In fact, let $X^{c}$ denote the set of continuous complex-valued functions on two real variables $x$ and $y$ satisfying

i) $f(x, y+1)=f(x, y)$, and

ii) $f(x+1, y)=\mathrm{e}^{-2 \pi i c y} f(x, y)$.

Viewing the elements of $C\left(\mathrm{~T}^{2}\right)$ as periodic functions on two real variables, it is easy to check that $X^{c}$ is a $C\left(T^{2}\right)$-bimodule, under pointwise multiplication. If we now set $\langle f, g\rangle_{L}=f \bar{g},\langle f, g\rangle_{R}=\bar{f} g$, then $X^{c}$ becomes a Hilbert bimodule.

In general, if $X$ is a Hilbert bimodule over a $C^{*}$-algebra $A$ and $\alpha$ is an automorphism of $A$, we denote, as in [2], by $X_{\alpha}$ the Hilbert bimodule over $A$ that agrees with $X$ as a left Hilbert module but is equipped with the right Hilbert module structure given by

$$
\begin{aligned}
x \cdot a & =x \alpha(a), \quad \text { for } x \in X, a \in A, \\
\langle x, y\rangle_{R}^{M_{\alpha}} & =\alpha^{-1}\left(\langle x, y\rangle_{R}\right), \quad \text { for } x, y \in X .
\end{aligned}
$$

Now, given real parameters $\mu$ and $\nu$, consider the automorphism $\alpha_{\mu, \nu}$ of $C\left(\mathrm{~T}^{2}\right)$ given by

$$
\left.\alpha_{\mu, v}(f)\right|_{(x, y)}=f(x+2 \mu, y+2 v) .
$$

It was shown in [1] and [2, Section 2] that $D_{\mu, v}^{c}$ is isomorphic to the crossed product of $C\left(\mathrm{~T}^{2}\right)$ by $X_{\alpha_{\mu, v}}^{c}$.

As in our earlier examples, we will show that $D_{\mu, v}^{c}$ can be described as a deformation of $C\left(M^{c}\right)$ relative to a certain deformation data.

Given $(x, y, z)$ in the Heisenberg group $H$, we denote its class in $H / G^{c}$ by $[x, y, z]$. Let $\mu$ and $v$ be fixed real numbers and consider the map $\phi$, from $\mathrm{R}^{2}$ into $H$, given by

$$
\begin{aligned}
\phi(a, b) & :=\exp \left(a\left(\begin{array}{ccc}
0 & 0 & 1 / c \\
0 & 0 & 0 \\
0 & 0 & 0
\end{array}\right)+b\left(\begin{array}{ccc}
0 & 2 v & 0 \\
0 & 0 & 2 \mu \\
0 & 0 & 0
\end{array}\right)\right) \\
& =\left(\begin{array}{ccc}
1 & 2 b v & 2 b^{2} \mu v+a / c \\
0 & 1 & 2 b \mu \\
0 & 0 & 1
\end{array}\right) .
\end{aligned}
$$

Since the two summands being exponentiated commute, one sees that $\phi$ is a group homomorphism, yielding an action of $\mathrm{R}^{2}$ on $H$, by left multiplication. 
This action, which obviously commutes with the right action of $G^{c}$ on $H$, drops to the quotient, producing the following action of $\mathrm{R}^{2}$ on $M^{c}$ :

$$
\phi_{(a, b)}[x, y, z]=\left[x+2 b \mu, y+2 b v, z+2 b v x+2 b^{2} \mu v+a / c\right],
$$

for $(a, b) \in \mathrm{R}^{2}$ and $[x, y, z] \in M^{c}$.

If we now let $\left(Z, \gamma, \theta^{\hbar}\right)$ be the deformation data given by $\phi$, as in section 5 , the gauge action $\gamma$, seen, as before, as an action of the circle group, will be $\gamma_{\mathrm{e}^{2 \pi i t}}([x, y, z])=[x, y, z+t / c]$, while the deforming actions $\theta^{\hbar}$ of $Z$ on $M^{c}$ are given by iterating the diffeomorphism

$\theta^{\hbar}([x, y, z])=\left[x+2 \hbar \mu, y+2 \hbar v, z+2 \hbar v x+2 \hbar^{2} \mu \nu\right], \quad$ for $[x, y, z] \in M^{c}$.

Let us assume that $\hbar=1$ or, what amounts to the same, that $\mu$ and $v$ are replaced, respectively, by $\hbar \mu$ and $\hbar \nu$. So we denote the $\theta^{\hbar}$ above simply by $\theta$. For each integer $k$ let $B_{k}$ be the $k$-spectral subspace for the gauge action $\gamma$ on $C\left(M^{c}\right)$. In particular, the fixed-point algebra $B_{0}$, coincides with the algebra of continuous functions on the quotient $M^{c} / S^{1}$. It is a simple task to verify that the map $[x, y, z] \in M^{c} \longmapsto\left(\mathrm{e}^{2 \pi i x}, \mathrm{e}^{2 \pi i y}\right) \in \mathrm{T}^{2}$ drops to a homeomorphism from $M^{c} / S^{1}$ to the 2-torus $\mathrm{T}^{2}$. In other words, $B_{0}$ is isomorphic to $C\left(\mathrm{~T}^{2}\right)$.

In general, for each $k$ in Z, the $k$-spectral subspace $B_{k}$ is given by the set of functions $f: M^{c} \rightarrow C$ satisfying $\gamma_{\lambda}(f)=\lambda^{k} f$, for $\lambda$ in $S^{1}$ or, equivalently, $f[x, y, z+t / c]=\mathrm{e}^{2 \pi i k t} f[x, y, z]$.

This reflects the fact that $\gamma$ is the dual action of $C\left(M^{c}\right)$, when the latter is viewed as a Hilbert-bimodule crossed product [1]. Now, this implies that $f[x, y, z]=\mathrm{e}^{2 \pi i k c z} f[x, y, 0]$. So $f$ is determined by its values on the elements $[x, y, 0]$. This suggests defining, for each such $f$, the function $g(x, y):=$ $f[x, y, 0]$. Since $(x, y, 0)(0,1,0)=(x, y+1,0)$, we see that $g$ is periodic in its second variable. Moreover, since $(x, y, 0)(1,0,0)=(x+1, y, y)$, we have that

$$
\begin{aligned}
g(x, y)=f[x, y, 0]=f[x+1, y, y] & =\mathrm{e}^{2 \pi i k c y} f[x+1, y, 0] \\
& =\mathrm{e}^{2 \pi i k c y} g(x+1, y) .
\end{aligned}
$$

Summarizing, we have

i) $g(x, y+1)=g(x, y)$, and

ii) $g(x+1, y)=\mathrm{e}^{-2 \pi i k c y} g(x, y)$,

which the reader should compare with the equations defining the Hilbert bimodule $X^{c}$, earlier in this section. Conversely, given any continuous function $g: \mathbf{R}^{2} \rightarrow \mathrm{C}$ satisfying (i) and (ii) above, one may define $f[x, y, z]=$ $\mathrm{e}^{2 \pi i k c z} g(x, y)$, and, after verifying that $f$ is indeed well defined, show that $f \in B_{k}$. 
We next observe that the gauge action on $M^{c}$ is semi-saturated, that is, $C\left(M^{c}\right)$ is generated, as a $C^{*}$-algebra, by $B_{0}$ and $B_{1}$. This follows from the fact that $C\left(M^{c}\right)$ is a Hilbert-bimodule crossed product [1, Theorem 3.1] (see also $[6,4.1,4.8]$ and $[7,6.2])$.

THEOREM 8.3. For every positive integer $c$, and real numbers $\mu$ and $\nu$, we have that $C\left(M^{c}\right)_{\gamma}^{\theta}$ is isomorphic to $D_{\mu, v}^{c}$.

Proof. By 2.3 we have that $\mathscr{B}^{\theta}$ is also semi-saturated and hence, by [1], in conjunction with [7, 4.2 and 4.7], we conclude that $C\left(M^{c}\right)_{\gamma}^{\theta}$, that is $C^{*}\left(\mathscr{B}^{\theta}\right)$, is given by the Hilbert bimodule crossed product $B_{0} \rtimes_{B_{1}}$ Z. It is important to stress that the $B_{0}-$ Hilbert bimodule structure of $B_{1}$ we are referring to, is that coming from the operations of $C^{*}\left(\mathscr{B}^{\theta}\right)$, that is, the deformed bundle operations $\times$ and ${ }^{\diamond}$ of $\mathscr{B}^{\theta}$. To make this more explicit, let $a \in B_{0}$ and $b, c \in B_{1}$, which we may assume are given by $a[x, y, z]=f(x, y), b[x, y, z]=\mathrm{e}^{2 \pi i c z} g(x, y)$, and $c[x, y, z]=\mathrm{e}^{2 \pi i c z} h(x, y)$, where $f$ is periodic and both $g$ and $h$ satisfy the conditions (i) and (ii) above for $k=1$. The reader may then verify that

$$
\begin{aligned}
a \times b[x, y, z] & =\mathrm{e}^{2 \pi i c z} f(x, y) g(x, y), \\
b \times a[x, y, z] & =\mathrm{e}^{2 \pi i c z} g(x, y) f(x+2 \mu, y+2 v), \\
b^{\diamond} \times c[x, y, z] & =\overline{g(x-2 \mu, y-2 \nu)} h(x-2 \mu, y-2 \nu), \\
b \times c^{\diamond}[x, y, z] & =g(x, y) \overline{h(x, y)} .
\end{aligned}
$$

These formulas tell us that the pair $\left(B_{0}, B_{1}\right)$ is isomorphic to $\left(C\left(\mathrm{~T}^{2}\right), X_{\alpha_{\mu, v}}^{c}\right)$ as a Hilbert bimodule. Hence, since $C\left(M^{c}\right)_{\gamma}^{\theta}=B_{0} \rtimes_{B_{1}} \mathrm{Z}$ and $D_{\mu, v}^{c}=C\left(\mathrm{~T}^{2}\right) \rtimes_{X_{\alpha \mu, v}^{c}} \mathrm{Z}$, we conclude that $C\left(M^{c}\right)_{\gamma}^{\theta} \simeq D_{\mu, \nu}^{c}$.

Let us now compute the differential operators $\partial_{x}$ and $\partial_{y}$, as in section 5 , arising from the action $\phi$ of $\mathrm{R}^{2}$ on $M^{c}$, which we denote by $\partial_{a}$ and $\partial_{b}$, respectively. For a smooth function $f$ on $M^{c}$, we have

$$
\partial_{a}(f)[x, y, z]=\left.\frac{d}{d a}(f[x, y, z+a / c])\right|_{a=0}=c^{-1} \partial_{3}(f)[x, y, z],
$$

while

$$
\begin{aligned}
\partial_{b}(f)[x, y, z] & =\left.\frac{d}{d b}\left(f\left[x+2 b \mu, y+2 b v, z+2 b^{2} \mu \nu+2 b v x\right]\right)\right|_{b=0} \\
& =\left(2 \mu \partial_{1}(f)+2 v \partial_{2}(f)+2 v x \partial_{3}(f)\right)[x, y, z]
\end{aligned}
$$

where $\partial_{1}, \partial_{2}$ and $\partial_{3}$ correspond to the partial differentiation operators for the standard coordinates on $\mathrm{R}^{3}$. 
The Poisson bracket on $M^{c}$ becomes

$\{\cdot, \cdot\}=\partial_{a} \wedge \partial_{b}=c^{-1} \partial_{3} \wedge\left(2 \mu \partial_{1}+2 v \partial_{2}+2 v x \partial_{3}\right)=2 c^{-1} \partial_{3} \wedge\left(\mu \partial_{1}+v \partial_{2}\right)$, which, up to a multiplicative factor, is the Poisson bracket considered in section 2 of [16]. We may therefore deduce from 5.7 and 8.3, one of the main results of [16]:

THEOREM 8.4. The family $\left\{D_{\hbar \mu, \hbar \nu}^{c}\right\}_{\hbar \in \mathrm{R}}$ forms a strict deformation quantization of $M^{c}$ in the direction of the Poisson bracket $2 c^{-1} \partial_{3} \wedge\left(\mu \partial_{1}+v \partial_{2}\right)$.

\section{REFERENCES}

1. Abadie, B., Eilers, S. and Exel, R., Morita equivalence for crossed products by Hilbert bimodules, Trans. Amer. Math. Soc. 350 (1998), 3043-3054.

2. Abadie, B. and Exel, R., Hilbert $C^{*}$-bimodules over commutative $C^{*}$-algebras and an isomorphism condition for quantum Heisenberg manifolds, Rev. Math. Phys. 9 (1997), 411423.

3. Bratteli, O., Derivations, Dissipations and Group Actions on $C^{*}$-Algebras, Lecture Notes in Math. 1229, 1986.

4. Dixmier, J., $C^{*}$-Algebras, North Holland, 1982.

5. Exel, R., The soft torus and applications to almost commuting matrices, Pacific J. Math., 160 (1993), 207-217.

6. Exel, R., Circle actions on $C^{*}$-algebras, partial automorphisms and a generalized PimsnerVoiculescu exact sequence, J. Funct. Anal. 122 (1994), 361-401.

7. Exel, R., Amenability for Fell bundles, J. Reine Angew. Math. 492 (1997), 41-73.

8. Fell, J. M. G. and Doran, R. S., Representations of ${ }^{*}$-Algebras, Locally Compact Groups, and Banach * -algebraic Bundles, Pure and Applied Mathematics 125 and 126, Academic Press, 1988.

9. Jørgensen, P. E. T., Schmitt, L. M. and Werner, R. F., q-canonical commutation relations and stability of the Cuntz algebra, Pacific J. Math. 165 (1994), 131-151.

10. Matsumoto, K., Noncommutative three-dimensional spheres, Japan. J. Math. (N.S.) 17 (1991), 333-356.

11. Matsumoto, K., Noncommutative three-dimensional spheres. II. Noncommutative Hopf fibering, Yokohama Math. J. 38 (1991), 103-111.

12. Matsumoto, K. and Tomiyama, J., Noncommutative lens spaces, J. Math. Soc. Japan 44 (1992), 13-41.

13. Ng, C. -K., Reduced Cross-sectional $C^{*}$-algebras of $C^{*}$-algebraic bundles and Coactions, preprint, Oxford University, 1996.

14. Rieffel, M. A., $C^{*}$-algebras associated with irrational rotations, Pacific J. Math. 93 (1981), 415-429.

15. Rieffel, M. A., Continuous fields of $C^{*}$-algebras coming from group cocycles and actions, Math. Ann. 283 (1989), 631-643.

16. Rieffel, M. A., Deformation quantization of Heisenberg manifolds, Comm. Math. Phys. 122 (1989), 531-562.

17. Rieffel, M. A., Deformation quantization for actions of $\mathbf{R}^{d}$, Mem. Amer. Math. Soc. 106 (1993), 93 pp.

18. Rieffel, M. A., Quantization and $C^{*}$-algebras, Contemp. Math. 167 (1994), 66-97. 
19. Woronowicz, S. L., Twisted $S U_{2}$ groups. An example of a non-commutative differential calculus, Publ. Res. Inst. Math. Sci. 23 (1987), 117-181.

20. Zeller-Meier, G., Produits croisés d'une $C^{*}$-algèbre par un groupe d'automorphismes, J. Math. Pures Appl. 47 (1968), 101-239.

CENTRO DE MATEMÁTICAS

FACULTAD DE CIENCIAS

IGUÁ 4225

CP 11400, MONTEVIDEO

URUGUAY

E-mail: abadie@cmat.edu.uy
DEPARTAMENTO DE MATEMÁTICA

UNIVERSIDADE DE SÃO PAULO

RUA DO MATÃO, 1010

05508-900 SÃO PAULO

BRAZIL

E-mail: exel@ime.usp.br 